\title{
Ratio Correction Factor and Phase Displacement at Arbitrary Burdens in Instrument Transformers
}

\author{
Jae Kap Jung ${ }^{\dagger}$, Kyu-Tae Kim* and Young Seob Lee**
}

\begin{abstract}
Ratio correction factor and phase displacement of instrument transformers vary with the values of the external burden connected to the secondary of the transformers. Using the values measured at two burdens of the instrument transformers, the ratio correction factor and phase displacement at arbitrary burdens are theoretically obtained without any additional measurement. This is based on a theoretical analysis of the equivalent circuits of the instrument transformers. The validity of the methods was verified by comparing the theoretical value with measured value for several instrument transformers with different accuracy.
\end{abstract}

Keywords: Instrument transformer, Ratio error, Ratio correction factor, Phase displacement, Burden, Equivalent circuit, Expanded uncertainty

\section{Introduction}

Voltage transformers (VTs) and current transformers (CTs) are normally used in the power industry for high voltage, heavy current, and power loss measurements [1-3]. The method most widely used to calibrate the transformers is to compare the transformer under test with a reference transformer that has the same nominal ratio and higher accuracy $[4,5]$. According to the International Electrotechnical Commission (IEC) [6] and American National Standard Requirements for Instrument Transformers (ANSI) [7], the ratio correction factor and phase displacement of an instrument transformer under test should be measured by connecting an external burden to the secondary of the transformer under test. The measurements of ratio correction factor and phase displacement of the instrument transformers at two different burdens are required. However, the values of burden required in IEC are different from that in ANSI.

The users in industry are usually using their instrument transformers at the different burden conditions compared with the burdens calibrated according to requirement of IEC or ANSI. Therefore, it is very useful that the ratio correction factor and phase displacement of instrument transformer are obtained at the real burden complying with the environment of industry without any additional measurements.

In this study, we have developed the methods for obtaining the ratio correction factor and phase displacement at arbitrary any burdens without any additional accuracy

\footnotetext{
$\dagger$ Corresponding Author: Korea Research Institute of Standards and Science, Korea. (jkjung@kriss.re.kr)

* Korea Research Institute of Standards and Science, Korea. (ktkim@kriss.re.kr)

** Korea University of Science and Technology, Korea. (yshaha524@naver.com) Received: April 13, 2015; Accepted: March 31, 2016
}

measurement, from the ratio correction factor and phase displacement of instrument transformer measured at two different burdens according to IEC or ANSI. This burden effects are based on a theoretical analysis of the equivalent circuits of the VT and CT. The validity of this method was verified by comparing the theoretical value for several instrument transformers with measured value.

\section{Burden Effects on VT}

\subsection{Calculation of ratio correction factor and phase displacement at arbitrary burdens}

An equivalent circuit for a VT with a zero burden is shown in Fig. 1 [8]. $Z_{0}$ in Fig. 1 is the leakage output impedance of the secondary of the VT:

$$
Z_{0}=R_{0}+j X_{0}
$$

The complex ratio of the primary voltage vector $\left(V_{p}\right)$ to the secondary voltage vector $\left(V_{s}\right)$ of the VT with high accuracy at a zero burden is given by $[8,9]$.

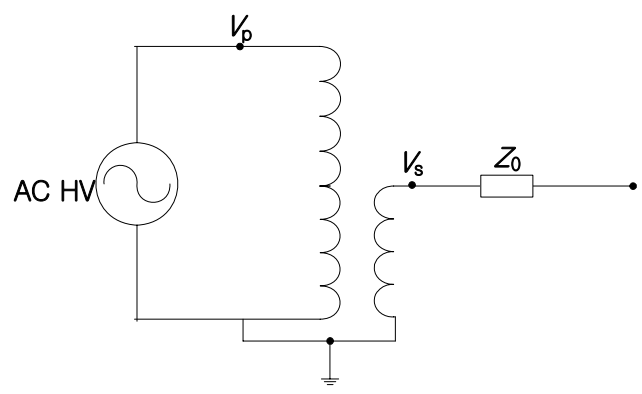

Fig. 1. Equivalent circuit for a VT with a zero burden 


$$
\begin{aligned}
\frac{V_{p}}{V_{s}} & =N_{V T} R C F_{0} e^{-j \beta_{0}} \cong N_{V T}\left(1-\alpha_{0}-j \beta_{0}\right) \\
& =N_{V T}\left(R C F_{0}-j \beta_{0}\right)
\end{aligned}
$$

In Eq. (2), $N_{\mathrm{VT}}$ is the rated transformation ratio of VT. $\alpha_{0}$ is the ratio error at a zero burden, and $\beta_{0}$ is the phase displacement between the secondary voltage vector and the primary voltage vector at a zero burden, defined as $\beta_{0} \equiv \beta_{s}-\beta_{p}$. The $R C F_{0}$ is the ratio correction factor at a zero burden, defined as the actual transformation ratio $\left(N_{a}\right)$ divided by the rated transformation ratio $\left(N_{V T}\right)$. The relationship between $R C F_{0}$ and $\alpha_{0}$ is as follows;

$$
R C F_{0} \equiv 1-\alpha_{0}
$$

An equivalent circuit for the VT with an external burden, $Z_{b}$, is shown in Fig. 2 [8]. The VT burden consists of a serial connection of the resistance and the inductor, expressed as:

$$
Z_{b}=R_{b}+j X_{b}
$$

In a similar manner to Eq. (2), the complex ratio of the primary voltage vector $\left(V_{p}\right)$ to the secondary voltage vector $\left(V_{b}\right)$ of a VT with high accuracy in the presence of an external burden is:

$$
\begin{aligned}
\frac{V_{p}}{V_{b}} & =N_{V T} R C F_{b} e^{-j \beta_{b}} \cong N_{V T}\left(1-\alpha_{b}-j \beta_{b}\right) \\
& =N_{V T}\left(R C F_{b}-j \beta_{b}\right)
\end{aligned}
$$

where $\alpha_{b}$ is the ratio error with the external burden, and $\beta_{b}$ is the phase displacement between the secondary voltage vector and the primary voltage vector with the external burden. $R C F_{b}$ is the ratio correction factor with the external burden, written as $R C F_{b} \equiv 1-\alpha_{b}$. The ratio error and the phase displacement of the VT arise from the secondary burden, the leakage inductance of the primary and secondary, the winding resistance of the primary and secondary, the magnetizing current, and the core loss $[10,11]$.

Equating the currents passing through $Z_{0}$ and $Z_{b}$, as shown in Fig. 2, we obtain the following equation.

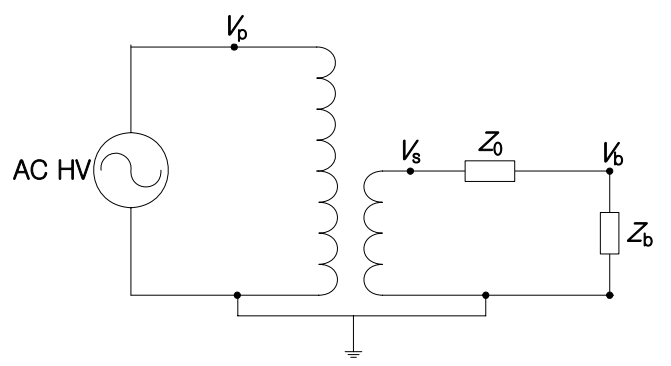

Fig. 2. Equivalent circuit for a VT with burden, $Z_{b}$

$$
\frac{V_{s}-V_{b}}{Z_{0}}=\frac{V_{b}}{Z_{b}}
$$

This can be rewritten as:

$$
\frac{V_{p}}{V_{b}}=\frac{V_{p}}{V_{s}}\left(1+\frac{Z_{0}}{Z_{b}}\right)
$$

Eq. (7) can be changed into Eq. (8) using Eqs. (1), (2), (4) and (5) as:

$$
R C F_{b}-j \beta_{b}=\left(R C F_{0}-j \beta_{0}\right)\left(1+\frac{R_{0}+j X_{0}}{R_{b}+j X_{b}}\right) .
$$

By taking the real part of Eq. (8), we can obtain the ratio correction factor with the external burden as:

$$
R C F_{b}=R C F_{0}\left(1+G_{b} R_{0}-B_{b} X_{0}\right)+\beta_{0}\left(G_{b} X_{0}+B_{b} R_{0}\right),
$$

where $G_{b}$ and $B_{b}$ are defined as follows.

$$
\begin{aligned}
G_{b} & \equiv \frac{R_{b}}{R_{b}{ }^{2}+X_{b}^{2}}: \text { conductance of the VT burden } \\
B_{b} & \equiv-\frac{X_{b}}{R_{b}{ }^{2}+X_{b}^{2}}: \text { susceptance of the VT burden }
\end{aligned}
$$

In a similar manner to Eq. (9), we can obtain the phase displacement with the external burden by taking the imaginary part of Eq. (8):

$$
\beta_{b}=\beta_{0}-R C F_{0}\left(G_{b} X_{0}+B_{b} R_{0}\right)+\beta_{0}\left(G_{b} R_{0}-B_{b} X_{0}\right)
$$

The leakage output impedance, $Z_{0}$, of the secondary of the VT is written by substituting subscript $t$ for $b$ in Eq. (7) as follows;

$$
Z_{0}=Z_{t}\left(\frac{V_{p} / V_{t}}{V_{p} / V_{s}}-1\right)=Z_{t}\left[\frac{R C F_{t} e^{-j\left(\beta_{t}-\beta_{0}\right)}-R C F_{0}}{R C F_{0}}\right]
$$

$\beta_{t}$ and $\beta_{0}$ is less than $10^{-3}$ order in Eq. (11), thus the quadratic and higher order terms in the exponential expansion of $e^{-j\left(\beta_{t}-\beta_{0}\right)}$ are less than $1 \times 10^{-6}$, which is neglected. Therefore, the resistance $\left(R_{0}\right)$ and reactance $\left(X_{0}\right)$ of $Z_{0}$ are given by as follows;

$$
\begin{gathered}
R_{0}=\left[\frac{\left(R C F_{t}-R C F_{0}\right) R_{t}+R C F_{t}\left(\beta_{t}-\beta_{0}\right) X_{t}}{R C F_{0}}\right] \\
X_{0}=\left[\frac{-R C F_{t}\left(\beta_{t}-\beta_{0}\right) R_{t}+\left(R C F_{t}-R C F_{0}\right) X_{t}}{R C F_{0}}\right]
\end{gathered}
$$

By measuring the ratio correction factor/phase 
displacement at the zero, $\mathrm{t}$ burden and impedance of $\mathrm{t}$ burden, we can obtain both $R_{0}$ and $X_{0}$ according to eqs. (12) and (13), respectively. Using values of $R_{0}$ and $X_{0}$, the ratio correction factor and phase displacement at arbitrary $b$ burden could be theoretically obtained without any additional accuracy experiment according to eqs. (9) and (10), respectively.

\subsection{Comparison between calculated and measured values for ratio correction factor and phase displacement at an arbitrary burden}

For study of burden effects, the ratio correction factor and phase displacement at four different burdens (zero, $t, b$ and $b$ ' burden) including zero burden are measured for three different types of VTs (VT 1, VT 2, and VT 3) with different accuracy. The VT 1, VT 2 and VT 3 have the accuracy of $0.005 \%, 0.02 \%$ and $0.2 \%$, respectively. The values of resistance/reactance of $t, b$ and $b$ ' burden are summarized in table 1 . Here $t, b$ and b' burden correspond to 4.87 VA with unity power factor, 5.05 VA with 0.80 lagging power factor and $15.02 \mathrm{VA}$ with 0.80 lagging power factor, respectively.

The measurement results of ratio correction factor/phase

Table 1. The values of resistance/reactance of $t, b$ and $b$ ' burdens

\begin{tabular}{c|c|c}
\hline Burden & Parameters & Value \\
\hline \multirow{2}{*}{ T burden } & $\mathrm{R}_{\mathrm{t}}$ & $2483 \Omega$ \\
& $\mathrm{X}_{\mathrm{t}}$ & $0 \Omega$ \\
\hline \multirow{2}{*}{ b burden } & $\mathrm{R}_{\mathrm{b}}$ & $1917.2 \Omega$ \\
& $\mathrm{X}_{\mathrm{b}}$ & $1437.9 \Omega$ \\
\hline \multirow{2}{*}{ B' burden } & $\mathrm{R}_{\mathrm{b}}$ & $645.4 \Omega$ \\
& $\mathrm{X}_{\mathrm{b}}$, & $481.9 \Omega$ \\
\hline
\end{tabular}

Table 2. Comparison between calculated and measured values of ratio correction factor/phase displacement for the voltage ratio of $3300 \mathrm{~V} / 110 \mathrm{~V}$ in three different type of VTs

\begin{tabular}{c|c|c|c|c}
\hline \multirow{2}{*}{ Burden } & \multirow{2}{*}{ Parameters } & \multicolumn{3}{|c}{ VT type } \\
\cline { 3 - 5 } & & VT 1 & VT 2 & VT 3 \\
\hline \multirow{2}{*}{ zero burden $^{*}$} & $R C F_{0}$ & 0.999890 & 0.999752 & 0.998114 \\
& $\beta_{0}[\mathrm{rad}]$ & -0.000006 & -0.000012 & 0.000227 \\
\hline \multirow{2}{*}{ t burden $^{*}$} & $R C F_{\mathrm{t}}$ & 1.000007 & 0.999835 & 0.998742 \\
& $\beta_{\mathrm{t}}[\mathrm{rad}]$ & -0.000047 & -0.000099 & 0.000020 \\
\hline \multirow{2}{*}{${\text { b } \text { burden }^{*}}^{*}$} & $R C F_{\mathrm{b}}$ & 1.000014 & 0.999873 & 0.998762 \\
& $\beta_{\mathrm{b}}[\mathrm{rad}]$ & 0.000032 & -0.000032 & 0.000448 \\
\hline \multirow{2}{*}{ b burden $^{+}$} & $R C F_{\mathrm{b}}$ & 1.000012 & 0.999875 & 0.998763 \\
& $\beta_{\mathrm{b}}[\mathrm{rad}]$ & 0.000033 & -0.000033 & 0.000446 \\
\hline \multirow{2}{*}{ difference $^{*}$} & $\Delta R C F_{\mathrm{b}}$ & 0.000002 & -0.000002 & -0.000001 \\
& $\Delta \beta_{\mathrm{b}}[\mathrm{rad}]$ & -0.000001 & 0.000001 & 0.000002 \\
\hline \multirow{2}{*}{$\mathrm{b}^{\prime}$ burden $^{*}$} & $R C F_{\mathrm{b}}$, & 1.000259 & 1.000116 & 1.000034 \\
& $\beta_{\mathrm{b}},[\mathrm{rad}]$ & 0.000105 & -0.000073 & 0.000887 \\
\hline \multirow{2}{*}{$\mathrm{b}^{\prime}$ burden $^{+}$} & $R C F_{\mathrm{b}}$, & 1.000255 & 1.000117 & 1.000045 \\
& $\beta_{\mathrm{b}},[\mathrm{rad}]$ & 0.000108 & -0.000074 & 0.000876 \\
\hline \multirow{2}{*}{ difference $^{*}$} & $\Delta R C F_{\mathrm{b}}$, & 0.000004 & -0.000001 & -0.000011 \\
& $\Delta \beta_{\mathrm{b}},[\mathrm{rad}]$ & -0.000003 & 0.000001 & 0.000011 \\
\hline
\end{tabular}

: measured value ${ }^{+}$: calculated value displacement at zero, $\mathrm{t}$ and $\mathrm{b}$ burdens for three different types of VTs are represented in the second, third and fourth row of table 2, respectively. From the errors measured at zero/t burdens and the impedance values of $t$ burden, the values of $R_{0}$ and $X_{0}$ for three different types of VTs using eqs. (12) and (13), respectively, are calculated as follows ;

$$
\begin{aligned}
& \mathrm{R}_{0}=0.29 \Omega, \mathrm{X}_{0}=0.10 \Omega \text { for VT } 1 \\
& \mathrm{R}_{0}=0.21 \Omega, \mathrm{X}_{0}=0.22 \Omega \text { for VT } 2 \\
& \mathrm{R}_{0}=1.56 \Omega, \mathrm{X}_{0}=0.51 \Omega \text { for VT } 3
\end{aligned}
$$

The ratio correction factor and phase displacement at arbitrary b burden are calculated using eqs. (9) and (10), respectively, as shown in the fifth row of table 2 . The differences between calculated and measured values at arbitrary $b$ burden are represented in the sixth row of table 2 , which are less than $2 \times 10^{-6}$ and shows good consistency. Meanwhile, the ratio correction factor and phase displacement are measured at arbitrary b' burden for three VTs, as shown in the seventh row of table 2 . The ratio correction factor and phase displacement at arbitrary b' burden according to eqs. (9) and (10) are calculated using $R_{0}$ and $X_{0}$ obtained already at zero and $\mathrm{t}$ burdens, as shown in the eighth row of table 2. The differences between calculated and measured values at arbitrary b' burden are represented in the last row of table 2 , which are less than $11 \times 10^{-6}$. The expanded uncertainty $(k=2)$ for the errors of the VTs was estimated to be within $30 \times 10^{-6}$ in ratio and 30 microrad in phase [12]. This implies that the measured values at arbitrary any burdens for VTs with different accuracy are good consistent with calculated value within the expanded uncertainty.

\section{Burden Effects on CT}

\subsection{Calculation of ratio correction factor and phase displacement at arbitrary burdens}

An equivalent circuit for a $\mathrm{CT}$ with an external burden, $Z_{\mathrm{b}}$, is shown in Fig. 3 [13-14]. The parameters shown in figure 3 are defined as follows:

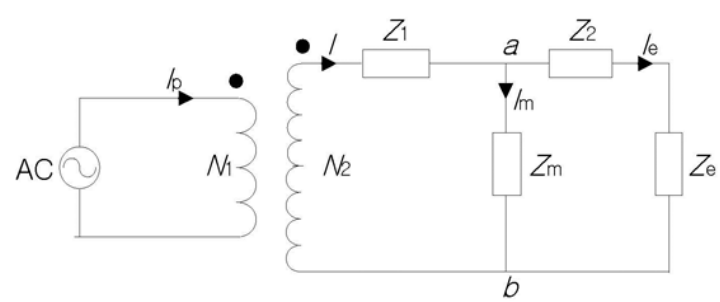

Fig. 3. Equivalent circuit for a CT with external burden, $Z_{\mathrm{e}}$.

$Z_{m}=R_{m}+j X_{m}$ : magnetizing impedance.

$Z_{1}=R_{1}+j X_{1}$ : primary leakage impedance.

$Z_{2}=R_{2}+j X_{2}$ : secondary leakage impedance. 
$Z_{e}=R_{e}+j X_{e}$ : impedance of external burden.

$N_{1}$ : number of primary windings.

$N_{2}$ : number of secondary windings.

$I_{\mathrm{p}} \quad$ : actual primary current.

$I_{\mathrm{e}} \quad$ : actual secondary current with an external burden.

$I \quad$ : ideal current with infinite magnetization impedance.

$I_{\mathrm{m}} \quad$ : magnetization current.

The complex ratio of the primary current vector $\left(I_{\mathrm{p}}\right)$ to the secondary current vector $\left(I_{\mathrm{e}}\right)$ in a CT with an external burden is given by [13].

$$
\begin{aligned}
\frac{I_{p}}{I_{e}} & =N_{C T}\left(1+\frac{Z_{2}+Z_{e}}{Z_{m}}\right) \cong N_{C T}\left(1-\varepsilon_{e}-j \delta_{e}\right) \\
& =N_{C T}(R C F-j \delta
\end{aligned}
$$

where $N_{C T}$ is the rated transformation ratio of CT. $\varepsilon_{e}$ is the ratio error with the external burden and $\delta_{e}$ is the phase displacement between the secondary current vector and the primary current vector with the external burden. $R C F_{e}$ is the ratio correction factor with the external burden, defined as $R C F_{e} \equiv 1-\varepsilon_{e}$.

By taking the real and imaginary parts of $\left(Z_{2}+Z_{\mathrm{e}}\right) / Z_{\mathrm{m}}$ in Eq. (14), we obtain the ratio correction factor $\left(R C F_{e}\right)$ and the phase displacement $\left(\delta_{e}\right)$, respectively, with the external burden as follows:

$$
\begin{gathered}
R C F_{e}=1+G_{m}\left(R_{2}+R_{e}\right)-B_{m}\left(X_{2}+X_{e}\right), \\
\delta_{e}=-B_{m}\left(R_{2}+R_{e}\right)-G_{m}\left(X_{2}+X_{e}\right),
\end{gathered}
$$

where $G_{m}=\frac{R_{m}}{R_{m}{ }^{2}+X_{m}{ }^{2}}$ is the conductive component of the excitation admittance, and $B_{m}=-\frac{X_{m}}{R_{m}{ }^{2}+X_{m}{ }^{2}}$ is the susceptive component of the excitation admittance.

When a non-reactive resistor with an AC-DC difference less than $10^{-5}[15]$, i. e., $\left(X_{\mathrm{e}} / R_{\mathrm{e}}\right)<10^{-5}$, is used as the external burden of the CT under test, then Eqs. (15) and (16) become:

$$
\begin{aligned}
& R C F_{e}=1+\left(G_{m} R_{2}-B_{m} X_{2}+G_{m} R_{e}\right)=\text { const. }+G_{m} R_{e} \\
& \delta_{e}=-B_{m} R_{2}-G_{m} X_{2}-B_{m} R_{e}=\text { const. }-B_{m} R_{e}
\end{aligned}
$$

Both the ratio correction factor $\left(R C F_{e}\right)$ and the phase displacement $\left(\delta_{e}\right)$ of the CT under test in Eqs. (17) and (18) are proportional to the resistance of external burden, $R_{\mathrm{e}}$, because of the constant values of $G_{\mathrm{m}}, B_{\mathrm{m}}, R_{2}$, and $X_{2}$ for a fixed secondary current. By measuring $R C F_{e}$ and $\delta_{e}$ at two resistive burdens, the slopes in Eqs. (17) and (18) give $G_{\mathrm{m}}$ and $B_{\mathrm{m}}$, respectively. From values of $G_{\mathrm{m}}$ and $B_{\mathrm{m}}$ obtained from measurements of two resistive burdens, the ratio correction factor and phase displacement at arbitrary any e burden can be calculated according to Eqs. (15) and
(16), respectively, without any additional accuracy measurement.

\subsection{Comparison between calculated and measured values for ratio correction factor and phase displacement at an arbitrary burden}

The ratio correction factor and phase displacement at four different burdens are measured for three different types of CTs (CT 1, CT 2, and CT 3) with different accuracy. The CT 1, CT 2 and CT 3 have the accuracy of $0.001 \%, 0.01 \%$ and $0.2 \%$, respectively. The values of resistance/reactance of $R_{a}, R_{b}$, e and e' burden and secondary winding resistance $\left(R_{2}\right)$ for three different types of $\mathrm{CT}$ are summarized in table $3 . \mathrm{R}_{\mathrm{a}}$ and $\mathrm{R}_{\mathrm{b}}$ indicate resistive burden with unity power factor. The e and $e^{\text {' }}$ indicate burden composing as a serial connection of a resistance and inductance. In general, the primary leakage reactance $\left(X_{1}\right)$ in a $\mathrm{CT}$ is much larger than secondary leakage reactance $\left(X_{2}\right)$, and the secondary windings are next to the core in a single layer. Therefore, it is reasonable to neglect $X_{2}$ entirely $[10,16]$.

The measurement results of ratio correction factor/phase displacement at $R_{a}$ and $R_{b}$ resistive burdens for three types of CTs are represented in the second and third row of Table 4, respectively. From the ratio correction factor/phase displacement obtained at $\mathrm{R}_{\mathrm{a}}$ and $\mathrm{R}_{\mathrm{b}}$ resistive burdens, the values of $G_{\mathrm{m}}$ and $B_{\mathrm{m}}$ are calculated using eqs. (17) and (18), respectively. The calculated values of $G_{\mathrm{m}}$ and $B_{\mathrm{m}}$ for three different types of CTs are presented as follows ;

$$
\begin{aligned}
& G_{\mathrm{m}}=0.000001 \mathrm{~S}, B_{\mathrm{m}}=0.000001 \mathrm{~S} \text { for CT } 1 \\
& G_{\mathrm{m}}=0.000036 \mathrm{~S}, B_{\mathrm{m}}=-0.000114 \mathrm{~S} \text { for CT } 2 \\
& G_{\mathrm{m}}=0.000659 \mathrm{~S}, B_{\mathrm{m}}=-0.000657 \mathrm{~S} \text { for CT } 3
\end{aligned}
$$

The ratio correction factor and phase displacement are measured at arbitrary e burden for three CTs, as shown in the fourth row of table 4 . The ratio correction factor and phase displacement at arbitrary e burdens are calculated

Table 3. Resistance/reactance at four burdens $\left(R_{a}, R_{b}\right.$, e and e' burden) and secondary winding resistance $\left(R_{2}\right)$ for three different types of CTs.

\begin{tabular}{c|c|c|c|c}
\hline \multirow{2}{*}{ Burden } & \multirow{2}{*}{ Parameters } & \multicolumn{3}{|c}{ CT type (current ratio) } \\
\cline { 3 - 5 } & & $\begin{array}{c}\mathrm{CT} 1 \\
(100 \mathrm{~A} / 1 \mathrm{~A})\end{array}$ & $\begin{array}{c}\mathrm{CT} 2 \\
(100 \mathrm{~A} / 5 \mathrm{~A})\end{array}$ & $\begin{array}{c}\text { CT 3 } \\
(100 \mathrm{~A} / 5 \mathrm{~A})\end{array}$ \\
\hline $\mathrm{R}_{\mathrm{a}}$ burden & $\mathrm{R}_{\mathrm{a}}$ & $0.184 \Omega$ & $0.184 \Omega$ & $0.184 \Omega$ \\
\hline $\mathrm{R}_{\mathrm{b}}$ burden & $\mathrm{R}_{\mathrm{b}}$ & $1.082 \Omega$ & $0.682 \Omega$ & $0.682 \Omega$ \\
\hline \multirow{2}{*}{ e burden } & $\mathrm{R}_{\mathrm{e}}$ & $0.192 \Omega$ & $0.192 \Omega$ & $0.192 \Omega$ \\
& $\mathrm{X}_{\mathrm{e}}$ & $0.056 \Omega$ & $0.056 \Omega$ & $0.056 \Omega$ \\
\hline \multirow{2}{*}{ e' burden } & $\mathrm{R}_{\mathrm{e}}$ & $0.555 \Omega$ & $0.555 \Omega$ & $0.555 \Omega$ \\
& $\mathrm{X}_{\mathrm{e}}$, & $0.368 \Omega$ & $0.368 \Omega$ & $0.368 \Omega$ \\
\hline & $\mathrm{R}_{2}$ & $3.200 \Omega$ & $0.367 \Omega$ & $0.210 \Omega$ \\
\hline
\end{tabular}

CT $1: 5$ kA Tettex company CT, accuracy : $0.001 \%$, rated burden : 5 VA CT $2: 2$ kA Cte-tech company CT, accuracy : $0.01 \%$, rated burden : 5 VA CT $3: 1.5 \mathrm{kA}$ Yokogawa company CT, accuracy : $0.2 \%$, rated burden : $15 \mathrm{VA}$ 
Table 4. Comparison between calculated and measured values for ratio correction factor/ phase displacement for the current ratio of $100 \mathrm{~A} / 5 \mathrm{~A}$ and $100 \mathrm{~A} / 1 \mathrm{~A}$ in three different type of CTs.

\begin{tabular}{|c|c|c|c|c|}
\hline \multirow[b]{2}{*}{ Burden } & \multirow[b]{2}{*}{ Parameters } & \multicolumn{3}{|c|}{ CT type (current ratio) } \\
\hline & & $\begin{array}{c}\text { CT 1 } \\
(100 \mathrm{~A} / 1 \mathrm{~A}) \\
\end{array}$ & $\begin{array}{c}\text { CT } 2 \\
(100 \mathrm{~A} / 5 \mathrm{~A}) \\
\end{array}$ & $\begin{array}{c}\text { CT } 3 \\
(100 \mathrm{~A} / 5 \mathrm{~A}) \\
\end{array}$ \\
\hline $\mathrm{R}_{\mathrm{a}}$ burden ${ }^{*}$ & $\begin{array}{c}R C F_{\mathrm{a}} \\
\delta_{\mathrm{a}}[\mathrm{rad}]\end{array}$ & $\begin{array}{c}0.999997 \\
-0.000001 \\
\end{array}$ & $\begin{array}{l}1.000024 \\
0.000077\end{array}$ & $\begin{array}{l}1.000298 \\
0.000340\end{array}$ \\
\hline $\mathrm{R}_{\mathrm{b}}$ burden ${ }^{*}$ & $\begin{array}{c}R C F_{\mathrm{b}} \\
\delta_{\mathrm{b}}[\mathrm{rad}]\end{array}$ & $\begin{array}{c}0.999998 \\
-0.000002\end{array}$ & $\begin{array}{l}1.000042 \\
0.000134\end{array}$ & $\begin{array}{l}1.000626 \\
0.000667\end{array}$ \\
\hline e burden ${ }^{*}$ & $\begin{array}{c}R C F_{\mathrm{e}} \\
\delta_{\mathrm{e}}[\mathrm{rad}]\end{array}$ & $\begin{array}{r}0.999997 \\
-0.000001 \\
\end{array}$ & $\begin{array}{l}1.000032 \\
0.000076 \\
\end{array}$ & $\begin{array}{l}1.000351 \\
0.000311 \\
\end{array}$ \\
\hline e burden $^{+}$ & $\begin{array}{c}R C F_{\mathrm{e}} \\
\delta_{\mathrm{e}}[\mathrm{rad}]\end{array}$ & $\begin{array}{r}1.000003 \\
-0.000003 \\
\end{array}$ & $\begin{array}{l}1.000027 \\
0.000062 \\
\end{array}$ & $\begin{array}{l}1.000302 \\
0.000227 \\
\end{array}$ \\
\hline difference & $\begin{array}{c}\Delta R C F_{\mathrm{e}} \\
\Delta \beta_{\mathrm{e}}[\mathrm{rad}]\end{array}$ & $\begin{array}{c}-0.000006 \\
0.000002\end{array}$ & $\begin{array}{l}0.000005 \\
0.000014\end{array}$ & $\begin{array}{l}0.000049 \\
0.000084\end{array}$ \\
\hline e' burden" & $\begin{array}{c}R C F_{\mathrm{e}}, \\
\delta_{\mathrm{e}},[\mathrm{rad}]\end{array}$ & $\begin{array}{c}0.999997 \\
-0.000001\end{array}$ & $\begin{array}{l}1.000082 \\
0.000106\end{array}$ & $\begin{array}{l}1.000792 \\
0.000335\end{array}$ \\
\hline $\mathrm{e}^{\prime}$ burden ${ }^{+}$ & $\begin{array}{c}R C F_{\mathrm{e}}, \\
\delta_{\mathrm{e}},[\mathrm{rad}]\end{array}$ & $\begin{array}{c}1.000003 \\
-0.000004\end{array}$ & $\begin{array}{l}1.000075 \\
0.000092 \\
\end{array}$ & $\begin{array}{l}1.000746 \\
0.000260\end{array}$ \\
\hline difference & $\begin{array}{c}\Delta R C F_{\mathrm{e}^{\prime}} \\
\Delta \delta_{\mathrm{e}^{\prime}}[\mathrm{rad}]\end{array}$ & $\begin{array}{c}-0.000006 \\
0.000003\end{array}$ & $\begin{array}{l}0.000007 \\
0.000014\end{array}$ & $\begin{array}{l}0.000046 \\
0.000075\end{array}$ \\
\hline
\end{tabular}

: measured value ${ }^{+}$: calculated value

using eqs. (15) and (16), respectively. The calculated ratio correction factor and phase displacement at arbitrary e burden are presented in the fifth row of table 4. The differences between calculated and measured values at arbitrary e burden for three CTs are shown in the sixth row of table 4 , which are less than $14 \times 10^{-6}$ for CT 1 , CT 2 and $84 \times 10^{-6}$ for CT 3 . Meanwhile, the ratio correction factor and phase displacement are measured at arbitrary e' burden for three CTs, as shown in the seventh row of table 4. The ratio correction factor and phase displacement calculated at arbitrary e' burden using eqs. (15) and (16), respectively, are presented in the eighth row of table 4 . The differences between calculated and measured values at arbitrary e' burden for three CTs are shown in the last row of table 4 , which are less than $14 \times 10^{-6}$ for CT 1 , CT 2 and $75 \times 10^{-6}$ for CT 3.

The expanded uncertainty $(k=2)$ for the errors of the CTs was estimated to be within $30 \times 10^{-6}$ in ratio and 30 microrad in phase for the case of CT with higher accuracy [17]. This implies that the measured values at arbitrary any burdens for CT 1 and CT 2 are good consistent with calculated value within the expanded uncertainty. However, the relative large difference between calculated and measured values for CT 3 implies CT 3 with lower accuracy of $0.2 \%$ has lager measurement uncertainty than CT 1 and CT 2.

\section{Conclusions}

From the ratio correction factor and phase displacement measured at two different burdens, we have developed the methods for obtaining the ratio correction factor and phase displacement of instrument transformers at arbitrary any burdens without any additional accuracy measurement. The methods were applied the three VTs with the accuracy of $0.005 \%$ to $0.2 \%$ and three CTs with the accuracy of $0.001 \%$ to $0.2 \%$. The validity of these methods was verified by comparing the theoretical with experimental values. The calculated values for the several instrument transformers are consistent with measured values within the expanded uncertainty. For additional accuracy measurement of transformer under test in industry, they need a reference transformer with higher accuracy, which is only located in National Measurement Institute. Thus, it is more difficult to get the accuracy data precisely without reference transformer in industry itself. The proposed method could be possible it obtain the accuracy data at any arbitrary burden without the reference transformer in industry. This is also the merit of the proposed method. Consequently, this paper is a small contribution to understanding such burden effects on instrument transformers.

\section{Acknowledgements}

This work was supported by the National Research Council of Science \& Technology (NST) through Creative Agenda Project (CAP-11-5-KRISS)

\section{References}

[1] W. J. M. Moore, P. N. Miljanic, The Current Comparator, Peter Peregrinus Ltd., London, United Kingdom, 1988.

[2] P. N. Miljanic, E. So, An improved current-comparatorbased 1000 A transconductance amplifier for the insitu calibration of transformer loss measuring systems, IEEE Trans. Power Delivery 8 (3) (1993) 861-865.

[3] E. So, E. Hanique, In-situ calibration of high-voltage three-phase transformer loss measuring systems, IEEE Trans. Instrum. Meas. 50 (2) (2001) 422-425.

[4] A. H. M. Arnold, Current-transformer testing J. IEE 74 (449) (1934) 424-444.

[5] A. Braun, Determination of current transformer errors at primary currents up to $100000 \mathrm{~A}$, IEEE Trans. Instrum. Meas. IM-26 (3) (1977) 263-267.

[6] International Electrotechnical Commission 1999 Instrument Transformers: Part 2. Inductive Voltage Transformers IEC 60044-2 (Geneva: International Electrotechnical Commission) pp. 7. International Electrotechnical Commission 1996 Instrument Transformers: Part 1. Current Transformers IEC 60044-1 (Geneva: International Electrotechnical Commission) pp. 28

[7] American National Standard Requirements for 
Instrument Transformers, Am. Natl. Stands. Instit., (ANSI/IEEE) C57.13-1978 (reaffirmed, 1986), New York.

[8] W. E. Anderson, A calibration service for voltage transformers and high-voltage capacitors, NBS Measurement Services Special Publication 250-33 12-3, 1988.

[9] F. K. Harris, W. C. Sze, N. L. Kusters, O. Petersons, W. J. M. Moore, An international comparison of voltage-transformer calibration to $350 \mathrm{kV}$, IEEE Trans. Communications and Electronics 70 (1964) 18-19.

[10] P. J. Betts, Calculated temperature coefficients of transformers, Australia National Measurement Laboratory Technical Paper No. 4, 1977.

[11] J. K. Das, Determination of characteristics of a threewinding potential transformer and predetermination of errors, I. E. Journal-EL 51 (1970) 51-58.

[12] J. K. Jung, Calibration Procedure of Voltage Transformer in KRISS, Doc. No. C-13-2-0120-2006 (E), 2006.

[13] J. D. Ramboz, O. Petersons, A calibration service for current transformers, NIST Measurement Services, Special Publication 250-36, 1991.

[14] J. K. Jung, S. W. Kwon, S. H. Lee, Y. T. Park, A study on usefulness verification technique of the measurement system by the difference between calculated and experimental values of ratio error/phase angle error in current transformer, Trans. KIEE. 53C (2004) 213-217.

[15] Bishay catalog (www.vishay.com).

[16] H. W. Price, C. K. Duff, Effects of magnetic leakage in current transformers, University of Toronto Research Bulletin No. 2, 167-90, 1921

[17] J. K. Jung, Calibration Procedure of Current Transformer in KRISS, Doc. No. C-13-2-0100-2006 (E), 2006.

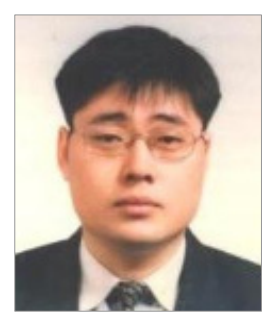

Jae Kap Jung He received the B.S. degree in physics and the M.S. and Ph.D. degrees in solid-state physics from Korea University, Seoul, Korea. He joined the Korea Research Institute of Standards and Science (KRISS), Daejeon, Korea, in 2001. He is currently a Principal Researcher with the Center for Energy Materials Metrology.

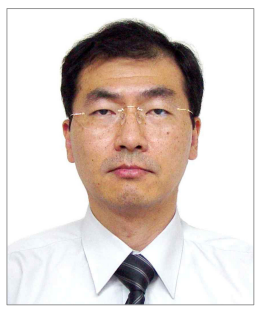

Kyu-Tae Kim received the B.S. degree in applied physics from Inha University, Incheon, South Korea, in 1983 and the M.S. and Ph.D. degree in physics from Korea Advanced Institute of Science and Technology (KAIST), Seoul, in 1985 and 1989, respectively. Since 1989, he has been with the Korea Research Institute of Standards and Science (KRISS), Daejeon where he has been working on the establishment of Josephson voltage standard, DC voltage ratio standard, ELF E-field calibration system, and DC high voltage/high current standard. He has been the leader in the field of Electricity and Magnetism at KRISS for 16 years, received the Presidential Citation from Korean government, in 2004, and the local technical program chair of the CPEM 2010, Daejeon.

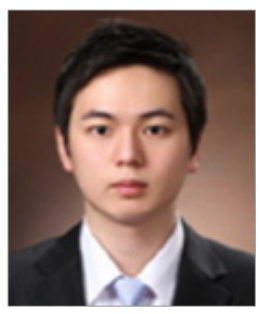

Young Seob Lee was born in 1988 in Korea. He received the B.S. degree in Kwangwoon University in Seoul. He is currently participating in the high voltage/high current research works in Korea Research Institute of Standards and Science (KRISS), Daejeon under M.S degree course with University of Science and Technology (UST). 